\title{
Research on Detectivity of Air-to-ground Missile Dual-mode Seeker
}

\author{
Hu Jian and Hu Fanjun \\ Department of Aviation Ammunition Engineering, the First Aeronautic Institute of the Air Force, \\ Xinyang, 464000, China \\ email:13523896492@163.com
}

\section{Keywords: Research, Dual-mode Seeker, Air-to-ground Missile, Detectivity}

\begin{abstract}
According to research on centralized detection theory of air-to-ground missile dual-mode seeker, the theory detecting threshold value is got by the method of likelihood ratio function. Based on quantitative analysis of air-to-ground missile dual-mode seeker detectivity, the relationship between $\mathrm{S} / \mathrm{N}$ and detection probability of single sensor and dual-mode seeker while radar and IR setting different detecting threshold value is found after simulation. The simulation results of instance proved the effectiveness and superiority of the proposed method.
\end{abstract}

\section{Introduction}

With the rapid development of scientific technology, the scale and structure of air-to-ground missile continue to become complicated. In the process of air-to-ground missile combating operation, more and more menace and challenge to single sensor seeker are confronted with [1]. The performance advantages of radar sensor and IR sensor, such as, target detecting, tracking and recognition, are comprehensive utilized in the radar and IR dual-mode seeker respectively. The dual-mode seeker can strengthen the existence of air-to-ground missile, heighten the reliability of seeker, widen the outlook in target detecting, raise the clarity in information processing and heighten the space distinguishability in target detection.

The detecting distance of radar sensor is far more than that of IR sensor. During the last guided stage, at first, the active radar sensor starts to work and search for target, once it detects the target, it guides the optical axle of IR sensor into the beam of radar antenna. Under the function of servo system, the optical axle of IR imagery sensor is kept in the same direction with the axle of radar antenna, then the IR imagery sensor searches for and captures target according to the indication of radar. The IR imagery sensor makes the further recognition and judgment for the beforehand locking target when it enters into the imagery scope of IR imagery sensor. The IR imagery sensor can search for, detect and track target independently when the radar sensor cannot work because of the radio silent and jamming [2] [4].

The multi-sensor system checking target is decomposed into the centralized and distributed detection [3]. The centralized detecting target is that the original data obtained by sensors are directly transmitted to detecting centre and processed so as to make the overall decision. Its advantage is that the information transmitted by sensors can be utilized as more as possible and obtained the optimum checking performance. The shortage is that the high calculating rate in detecting centre is required and the information processing clarity is not high.

According to research on centralized detection theory of air-to-ground missile dual-mode seeker, the quantitative assessment of air-to-ground missile dual-mode seeker detectivity is proposed, the influence factors on target detection of the dual-mode seeker are analyzed. Therefore, it has better function to further improve the target detectivity of the dual-mode seeker.

\section{Dual-mode Seeker Centralized Detecting Model}

In order to quantitative research on dual-mode seeker centralized detectivity, the following assumptions are appointed.

The detecting range of radar sensor is usually farther than that of IR imagery sensor, the dual-mode seeker detectivity is only discussed within the detecting range of IR imagery sensor. 
The IR imagery sensor has the "point" detectivity, that is to say, when the detected target size is far smaller than that of system scope on certain range, the system can detect the target as long as it has certain SNR.

It is assumed that both radar and IR imagery sensors are under circumstances of known signal and Gauss white noise [5] [6].

The target detection probability is single detection probability.

For selecting the theoretical detecting threshold, the radar/IR imagery sensor dual-mode seeker is the signal compound of radar sensor and IR imagery sensor, that is to say, the radar sensor and IR imagery sensor are installed in the same seeker, the two sensors work independently and have signal contact [2]. For example, the ground target has both radar and IR radiation features which are independently and irrelative. Two type signals of ground target can be obtained by means of radar/IR dual-mode seeker, the one is the target radar signal ur, ur $=\mathrm{sr}+\mathrm{nr}$, sr - the target radar signal, $\mathrm{nr}$ - the radar noise, the another is the target IR signal ui, ui=si+ni, si-the target IR signal, ni-the background IR noise.

There are four circumstances in dual-mode seeker receiving signals. $\mathrm{sr}=0$, $\mathrm{si}=0$, the dual-mode seeker receives no signal; $s r=0$, $s i \neq 0$, the dual-mode seeker only receives the IR signal; $s r \neq 0, s i=0$, the dual-mode seeker only receives the radar signal; $s r \neq 0, \quad s i \neq 0$, the dual-mode seeker receives both the radar and IR signals.

It is supposed that the judging rule adopted in the detecting centre is "or"rule when the dual-mode seeker centralized detects target, then the duality hypothesis checking for detecting target is stated as following.

s1-it is supposed that the target exists, s1=sr1

signal; $\mathrm{s} 0-\mathrm{it}$ is supposed that the target does not exist, $\mathrm{s} 0=\mathrm{sr} 0 \cap \mathrm{si} 0$, there is neither the radar signal nor the IR signal.

Because of being independent the radar signal and the IR signal, then

$\mathrm{P}(\mathrm{s} 1)=\mathrm{P}(\mathrm{sr} 1 \cup \mathrm{si} 1)=\mathrm{Psr} 1+\mathrm{Psi1}-\mathrm{Psr} 1 \mathrm{Psi1}$

$\mathrm{P}(\mathrm{s} 0)=\mathrm{P}(\mathrm{sr} 0 \cap \mathrm{si} 0)=(1-\mathrm{Psr} 1)(1-\mathrm{Psi} 1)$

The probability ratio is decided in accordance with the statistical signal duality checking theory.

$\Lambda(\mathrm{u})=\mathrm{P}(\mathrm{u} / \mathrm{s} 1) / \mathrm{P}(\mathrm{u} / \mathrm{s} 0)$

The criterion judging the target existing is shown as the following.

$\Lambda(\mathrm{u}) \geqslant \mathrm{P}(\mathrm{s} 0) / \mathrm{P}(\mathrm{s} 1)$

That is to say, $\Lambda(\mathrm{u}) \mathrm{P}(\mathrm{s} 1) / \mathrm{P}(\mathrm{s} 0) \geqslant 1$, there is the signal, i.e., the target exists; $\Lambda(\mathrm{u}) \mathrm{P}(\mathrm{s} 1) / \mathrm{P}(\mathrm{s} 0)<$ 1 , there is no signal, i.e., the target does not exist. So it is necessary to compute the probability ratio $\Lambda(\mathrm{u})$ in order to judge whether the target exists or not. It is supposed that the target signal $\mathrm{u}$ is received in the detecting centre, then $u$ can be stated as two dimension vector.

$\mathrm{u}=\{\mathrm{ur}, \mathrm{ui}\}$

Then its statistical property is ar, ai; or2, бi2.

$\operatorname{ar}=\frac{1}{\mathrm{~N}} \sum_{1}^{\mathrm{N}} \mathrm{E}$ (ur) , ai $=\frac{1}{\mathrm{~N}} \sum_{1}^{\mathrm{N}} \mathrm{E}$ (ui)

or2 $=\mathrm{E}[(\mathrm{ur}(\mathrm{N})-$ ar)2], $\sigma \mathrm{i} 2=\mathrm{E}[(\mathrm{ui}(\mathrm{N})-\mathrm{ai}) 2]$

It is defined a and $\sigma 2$ as following.

$\mathrm{a}=\mathrm{E}(\mathrm{u})=\min \{$ ar, ai $\}$

$\sigma 2=\mathrm{D}(\mathrm{u})=\max \{\sigma \mathrm{r} 2, \sigma \mathrm{i} 2\}$

$\mathrm{P}(\mathrm{u} / \mathrm{s} 0)$ - the probability density function of the mixed wave while there is no signal under the condition of the known signal and Gauss white noise. Its distribution rule is the same as that of the noise, that is to say,

$\mathrm{P}(\mathrm{u} / \mathrm{s} 0)=\frac{1}{\sqrt[3]{2 \pi \sigma}} \exp (-\mathrm{u} 2 / 2 \sigma 2)$

$\mathrm{P}(\mathrm{u} / \mathrm{s} 1)$ - the probability density function of the mixed wave while there is the signal under the condition of the known signal and Gauss white noise. Its distribution rule is the same as that of the noise, only the mean value is a, that is to say,

$$
\mathrm{P}(\mathrm{u} / \mathrm{s} 1)=\frac{1}{\sqrt{2 \pi \sigma}} \exp (-(\mathrm{u}-\mathrm{a}) 2 / 2 \sigma 2)
$$


Then the probability ratio is $\Lambda(\mathrm{u})$.

$\Lambda(\mathrm{u})=\mathrm{P}(\mathrm{u} / \mathrm{s} 1) / \mathrm{P}(\mathrm{u} / \mathrm{s} 0)=\exp ((-\mathrm{a} 2+2 \mathrm{ua}) / 2 \sigma 2)$

The judging criterion is transformed as following.

$\exp ((-\mathrm{a} 2+2 \mathrm{ua}) / 2 \sigma 2) \geqslant \mathrm{P}(\mathrm{s} 0) / \mathrm{P}(\mathrm{s} 1)$

Then, $\mathrm{u} \geqslant \sigma(\sigma \ln (\mathrm{P}(\mathrm{s} 0) / \mathrm{P}(\mathrm{s} 1)) / \mathrm{a}+\mathrm{a} / 2 \sigma)=\mathrm{u} 0$

Where, u0 - the threshold under the "or" rule. It is judged that the target exists while the mixed wave amplitude is more tnan u0, otherwise, the target does not exist.

The target existing priori probability is stated as following.

$\mathrm{P}(\mathrm{s} 0) / \mathrm{P}(\mathrm{s} 1)=((1-\mathrm{Psr} 1)(1-\mathrm{Psi1})) /($ Psr1+Psi1-Psr1Psi1)

\section{The Detection Probability of Dual-mode Seeker}

According to whether the mixed wave amplitude is more than the threshold or not, there may be four circumstances [7] [8].

s1 assumption is tenable, i. e., the target exists, the dual-mode seeker can detect the signal, then it is correct detecting and its probability is called the detection probability Pd.

s1 assumption is tenable, i. e., the target exists, the dual-mode seeker cannot detect the signal, then it is incorrect detecting and its probability is called the missing detection probability Pld.

s0 assumption is tenable, i. e., the target does not exist, the dual-mode seeker can detect the signal, then it is false alarm, its probability is called the false alarm probability Pfa.

s0 assumption is tenable, i. e., the target does not exist, the dual-mode seeker cannot detect the signal, then it is correct no detecting and its probability is called the no correct detection probability Pan.

While the target exists, $\alpha \mathrm{r} \neq 0$ or $\alpha \mathrm{i} \neq 0$, the probability density distribution of the radar and IR signal amplitude is submitted to zero-class Bessel function under the circumstances of both signal and noise being input into system at the same time.

$\mathrm{P}(\mathrm{ur} / \mathrm{s} 1)=\operatorname{urexp}(-(\mathrm{ur} 2+\alpha \mathrm{r} 2) / 2 \sigma \mathrm{r} 2) \mathrm{I} 0(\mathrm{ur} \alpha \mathrm{r} / \sigma \mathrm{r} 2) /$ or 2

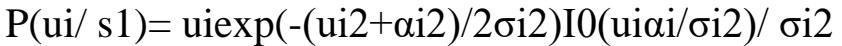

Where, ur-the radar signal and noise amplitude, ui-the IR signal and noise amplitude, $\alpha \mathrm{r}$ - the radar signal amplitude, $\alpha \mathrm{r}$ - the IR signal amplitude, $\sigma \mathrm{r}$ - the radar noise mean square bias, $\sigma \mathrm{i}$ - the IR noise mean square bias, IO(x) - the zero-class Bessel function of $\mathrm{x}$.

While the target does not exist, $\alpha \mathrm{r}=0=\alpha \mathrm{i}=0$, the probability density functions of the radar and IR noise amplitude are stated as following respectively.

$\mathrm{P}(\mathrm{ur} / \mathrm{s} 0)=\operatorname{urexp}(-\mathrm{ur} 2 / 2 \sigma \mathrm{r} 2) / \sigma \mathrm{r} 2$

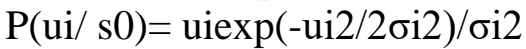

The dual-mode seeker probability density function of the signal and noise amplitude after receiver output is shown as following.

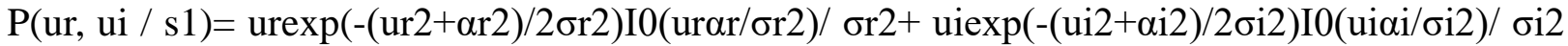

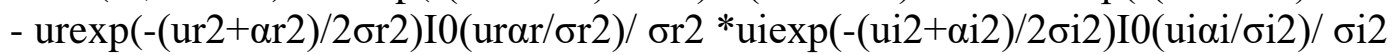

Then the detection probability is Pd.

$\mathrm{Pd}=\iint_{\text {urouio }}^{\infty} \mathrm{P}($ ur, ui $/$ s1) $\mathrm{durdui}=\operatorname{Pdr}(1-\mathrm{Pdi})+\operatorname{Pdi}(1-\mathrm{Pdr})+\mathrm{Pdr} \mathrm{Pdi}$

$\mathrm{Pdr}=\int_{u r 0}^{\infty} \mathrm{P}(\mathrm{ur} / \mathrm{s} 1) \mathrm{dur}=\mathrm{Q}(\mathrm{ur} / \sigma \mathrm{r}, \mathrm{ur} 0 / \sigma \mathrm{r})$

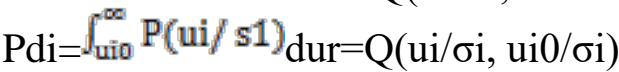

Where, ur0, ui0 - the radar and IR imagery sensor detection threshold respectively, and more over, ur0 $=\mathrm{ui} 0=\mathrm{u} 0, \mathrm{Q}(\mathrm{a}, \mathrm{b})$ - the Marcum-Q function.

The dual-mode seeker noise probability density function of the noise amplitude after receiver output is shown as following.

$\mathrm{P}($ ur, ui / s0) $=\operatorname{urexp}(-u r 2 / 2 \sigma r 2) / \sigma r 2 * u i e x p(-u i 2 / 2 \sigma i 2) / \sigma i 2$

Then the false alarm probability is Pfa.

Pfa $=\iint_{\text {urouio }}^{\infty} \mathrm{P}($ ur, ui $/ \mathrm{s} 0)$ durdui=PfarPfai 


$$
\begin{aligned}
& \text { Pfar }=\int_{\text {uro }}^{\infty} \mathrm{P}(\mathrm{ur} / \mathrm{s} 0) \mathrm{dur}=\exp (-\mathrm{u} 2 \mathrm{r} 0 / 2 \sigma 2 \mathrm{r}) \\
& \text { Pfai }=\int_{\text {uio }}^{\infty} \mathrm{P}(\mathrm{ui} / \mathrm{s} 0) \mathrm{dur}=\exp (-\mathrm{u} 2 \mathrm{i} 0 / 2 \sigma 2 \mathrm{i})
\end{aligned}
$$

\section{Computing Example Analysis}

In order to demonstrate the detectivity of air-to-ground missile dual-mode seeker, the following conditional parameters are selected. The signal to noise ratio of radar system is $(\mathrm{S} / \mathrm{N}) \mathrm{r}=\mathrm{ur} / \mathrm{\sigma r}$, and the IR system is $(\mathrm{S} / \mathrm{N}) \mathrm{i}=\mathrm{ui} / \sigma \mathrm{i}$. The dual-mode seeker detection probability is searched in circumstances of known permitted false alarm probability according to the Neyman-Pearson criterion [9] [10]. It is supposed that the dual-mode seeker target detection probability is more than $90 \%$ under condition of the false alarm probability being no more than $10-4$. The example is computed as following.

Example 1: $\mathrm{S} / \mathrm{N}=[0: 0.5: 10]$, ur0/ $\sigma \mathrm{r}=2.5, \mathrm{ui} 0 / \sigma \mathrm{i}=3.5$, The computing result of the total false alarm probability is $9.6 * 10-5$. The simulation results of the the detection probability are shown in Table 1.

Under the condition of satisfaction with the total false alarm probability requirement, the radar and IR imagery sensor needed SNR obtained the target detection probability $90 \%$ is $3.6 \mathrm{~dB}$ and 4.7dB respectively, the dual-mode seeker needed SNR is 3.2dB.

Tab.1 Simulation Result of Detection Probability

\begin{tabular}{|c|c|c|c|c|c|}
\hline $\mathrm{P}_{\mathrm{d}} / \mathrm{SNR}$ & 1 & 2 & 3 & 4 & 5 \\
\hline radar & 0.12 & 0.4 & 0.74 & 0.94 & 0.99 \\
\hline IR & 0.01 & 0.1 & 0.36 & 0.72 & 0.95 \\
\hline Dual-mode & 0.125 & 0.45 & 0.85 & 0.99 & 1.0 \\
\hline
\end{tabular}

Example 2: $\mathrm{S} / \mathrm{N}=[0: 0.5: 10]$, ur0/ $/ \mathrm{r}=5.0$, ui $/ \sigma \mathrm{i}=3.5$, The computing result of the total false alarm probability is $8.2 * 10-9$. The simulation results of the the detection probability are shown in Table 2 .

Tab.2 Simulation Result of Detection Probability

\begin{tabular}{|c|c|c|c|c|c|}
\hline $\mathrm{P}_{\mathrm{d}} / \mathrm{SNR}$ & 1 & 2 & 3 & 4 & 5 \\
\hline radar & 0.00 & 0.001 & 0.03 & 0.19 & 0.53 \\
\hline IR & 0.001 & 0.1 & 0.32 & 0.72 & 0.93 \\
\hline Dual-mode & 0.006 & 0.105 & 0.35 & 0.78 & 0.98 \\
\hline
\end{tabular}

Example 3: $\mathrm{S} / \mathrm{N}=[0: 0.5: 10]$, ur0/ $\sigma \mathrm{r}=2.5$, ui0/ $\sigma \mathrm{i}=4.0$, The computing result of the total false alarm probability is $1.5 * 10-5$. The simulation results of the the detection probability are shown in Table 3 .

Tab.3 Simulation Result of Detection Probability

\begin{tabular}{|c|c|c|c|c|c|}
\hline $\mathrm{P}_{\mathrm{d}} / \mathrm{SNR}$ & 1 & 2 & 3 & 4 & 5 \\
\hline radar & 0.12 & 0.4 & 0.73 & 0.94 & 0.99 \\
\hline IR & 0.00 & 0.04 & 0.18 & 0.55 & 0.85 \\
\hline Dual-mode & 0.125 & 0.45 & 0.79 & 0.96 & 1.0 \\
\hline
\end{tabular}

Under the condition of satisfaction with the total false alarm probability requirement, the radar and IR imagery sensor needed SNR obtained the target detection probability $90 \%$ is $3.6 \mathrm{~dB}$ and $5.2 \mathrm{~dB}$ respectively, the dual-mode seeker needed SNR is 3.4dB. The IR imagery sensor detection threshold should be raised while IR imagery sensor is disturbed and cannot work effectively, and the target detection probability is reduced relatively. The dual-mode seeker target detection probability is close to that of the radar sensor.

\section{Conclusion}

The relative superiorities of the multisensor detection and single sensor detection are quantitative analyzed based on the joint detection probability of air-to-ground missile dual-mode seeker.

The comprehensive detectivity of the dual-mode seeker is better than that of the single sensor in centralized detection, moreover, the dual-mode seeker ensure effective detection target while a 
certain sensor cannot be utilized or disturbed, so that the target detection continuity and effectiveness are realized.

The detection threshold should be raised while target cannot be detected effectively as a certain sensor is disturbed, and the target detection probability is reduced on the same SNR level.

The dual-mode seeker target detection probability can be kept stable when a certain SNR level is obtained. The SNR level decides the target detection difficulty.

\section{References}

[1] He You. Multisensor information fusion and application [M]. Beijing: Electronic Industry Press, 2006.

[2] Wang Chaoqun. Research on the joint detection probability of radar/IR imagery dual-mode seeker [J]. IR and Laser Engineering, pp. 221-225, March, 2003.

[3] Liu Longhe. Multisensor complex homing guidance technology [M]. Beijing: Defense Industry Press, 1998.

[4] Xin Tengda. Research on fuzzy sliding mode terminal guidance law with impact angle constraint for air-to-ground missile [J]. Tactical Missile Technology, pp. 61-69, May, 2015.

[5] Kada B. Arbitrary-order sliding-mode-based homing missile guidance for intercepting highly maneuverable targets [J]. Guidance, Control and Dynamics, 2014, 37(6):1999-2013.

[6] Liu Longhe. Complex ending guidance and performance analysis [J]. Systematic Engineering and Electronic Technology, 1997, 22 (3):4-7.

[7] Zhou Gang. Research on the target detection and identification probability of IR imagery system [J]. Infrared Technology, 2001, 23 (3):16-18.

[8] Chen Yuru. Anti-disturbance performance analysis of the radar/IR integrative detection system [J]. Infrared Technology, 2006, 28 (8):481-484.

[9] Zhou Di. Study of optimal sliding-mode guidance law [J]. Chinese Journal of Aeronautics, 1999, 12 (4):236-241.

[10]Qin Tian. A method for precision missile guidance with impact attitude angle constraint [J].Journal of Astronautics, 2012, 33 (5):570-576. 